\title{
Article \\ An Evaluation of Antibiotic Prescribing Practices in a Rural Refugee Settlement District in Uganda
}

\author{
Matua Bonniface ${ }^{1,2}$, Winnie Nambatya ${ }^{3}$ and Kalidi Rajab ${ }^{2,3, *}$ \\ 1 Department of Health, Yumbe District Local Government, 60811 Yumbe, Uganda; bonifacematua@yahoo.com \\ 2 East African Community Regional Center of Excellence for Vaccines, Immunizations and Health Supply \\ Chain, Kicukiro Campus, School of Public Health, University of Rwanda, Kigali, Rwanda \\ 3 Department of Pharmacy, Makerere University, University Rd, 10218 Kampala, Uganda; \\ wnambatya@chs.mak.ac.ug \\ * Correspondence: rkalidi@chs.mak.ac.ug; Tel.: +256-776798978
}

Citation: Bonniface, M.; Nambatya, W.; Rajab, K. An Evaluation of Antibiotic Prescribing Practices in a Rural Refugee Settlement District in Uganda. Antibiotics 2021, 10, 172. https://doi.org/10.3390/ antibiotics 10020172

Academic Editor: Akke Vellinga

Received: 31 December 2020

Accepted: 2 February 2021

Published: 9 February 2021

Publisher's Note: MDPI stays neutral with regard to jurisdictional claims in published maps and institutional affiliations.

Copyright: (c) 2021 by the authors. Licensee MDPI, Basel, Switzerland. This article is an open access article distributed under the terms and conditions of the Creative Commons Attribution (CC BY) license (https:// creativecommons.org/licenses/by/ $4.0 /)$.

\begin{abstract}
Ensuring access to effective antibiotics and rational prescribing of antibiotics are critical in reducing antibiotic resistance. In this study, we evaluated antibiotic prescribing practices in a rural district in Uganda. It was a cross-sectional study that involved a retrospective review of 500 outpatient prescriptions from five health facilities. The prescriptions were systematically sampled. World Health Organization core medicine use prescribing and facility indicators were used. Percentage of encounters with one or more antibiotics prescribed was $23 \%(10,402 / 45,160)$. The mean number of antibiotics per prescription was $1.3(669 / 500)$. About $27 \%(133 / 500)$ of the diagnoses and $42 \%$ $(155 / 367)$ of the prescriptions were noncompliant with the national treatment guidelines. Prescribing antibiotics for nonbacterial infections such as malaria 32\% (50/156) and noninfectious conditions such as dysmenorrhea and lumbago 15\% (23/156) and nonspecific diagnosis such as respiratory tract infection $40 \%$ (59/133) were considered noncompliant with the guidelines. On average, 68\% (51/75) of the antibiotics were available on the day of the visit. Inappropriate prescribing practices included excessive use of antibiotics and failure to diagnose and prescribe in compliance with treatment guidelines. There is a need to strengthen antibiotic use in the health facilities through setting up stewardship programs and interventions to promote adherence to national treatment guidelines.
\end{abstract}

Keywords: antibiotics; prescribing practices; availability

\section{Introduction}

Antibiotic resistance $(\mathrm{ABR})$ has recently been rising steadily worldwide and has reduced the ability of antibiotics to effectively control infectious diseases [1]. ABR causes 700,000 deaths annually across the globe, a number that is projected to increase to 10 million by the year 2050 if new interventions are not developed [2]. Data published from Uganda show an increase in the trends of ABR [3]. The prevalence of Methicillin-Resistant Staphylococcus Aureus (MRSA) varied from 2-50\%, while Extended Spectrum Beta-lactamase (ESBL) prevalence ranged from 10-75\% [4]. In addition, increasing resistance, ranging from 4-30\%, has been reported among Gram-negative enterobacteria against carbapenems, a last-line treatment, and a broad range of bacteria have still shown high levels of resistance (over 50\%) in many cases to commonly used antibiotics such as penicillin, tetracyclines, and co-trimoxazole [4]. In line with this, the United Nations (UN) and the World Health Organization (WHO) have called for actions to this public health threat [5].

The main driver of ABR is misuse of antibiotics, aggravated by other factors such as unrestricted access to antibiotics [6]. Common examples of irrational antibiotic use include incorrect diagnosis; prescribing of antibiotics for nonspecific conditions such as childhood diarrhea, mild, nonbacterial infection, upper respiratory tract infection, and simple malaria; polypharmacy; over-prescribing; excessive or unnecessary use of injections; improper use of antibiotics by patients; failure to prescribe in compliance with treatment guidelines; 
and the use of antibiotics as livestock food additives for growth promotion [7-11]. The consequences of such irrational antibiotic use include poor or limited quality of care, high cost of therapy, low availability, and increased incidence of adverse effects such as prolonged morbidity, mortality, drug toxicity, prolonged hospitalization, microbial antibiotic resistance, and the associated resistant infections [12-15].

Studies in Uganda show that most of the medicine use problems are caused by irrational prescribing practices [16]. However, most of these studies assessed prescribing practices generally or prescribing for specific health conditions such as upper respiratory tract infections, malaria, and simple diarrhea [17-21]. Little is known about prescribing practices for antibiotics in Yumbe, a refugee settlement and border district, and Uganda at large. We evaluated the prescribing practices of antibiotics in all the level 3 and 4 health facilities in Yumbe with the aim of providing information useful in designing interventions to improve appropriate use of antibiotics so as to achieve optimal clinical outcomes related to antibiotic use, minimizing toxicity and other adverse events, reducing the costs of health care for infections, and limiting the selection for antibiotic-resistant strains.

\section{Results}

In the review period, a total of 10,402 (23\%) prescriptions of the 45,160 prescriptions contained an antibiotic. The 500 sampled prescriptions of the 10,402 prescriptions with at least an antibiotic prescribed contained 669 antibiotics.

\subsection{Prescribing Practices}

The common diagnoses for which antibiotics were prescribed were respiratory tract infections $(117,23 \%)$, urinary tract infections $(84,17 \%)$, and malaria $(50,10 \%)$ (Table 1$)$.

Table 1. Showing diagnosis for which antibiotics were prescribed.

\begin{tabular}{ccc}
\hline Diagnosis & Frequency $(\boldsymbol{n = 5 0 0 )}$ & Percentage $\mathbf{( \% )}$ \\
\hline Respiratory tract infection & 117 & 23 \\
Urinary tract infection & 84 & 17 \\
Malaria & 50 & 10 \\
Gastroenteritis & 42 & 8 \\
Pelvic inflammatory disease & 29 & 6 \\
Peptic ulcer disease & 26 & 5 \\
Bacterial infection/septicemia & 19 & 4 \\
Helminthiasis & 16 & 3 \\
Otitis media & 11 & 2 \\
Septic wound/abscess & 9 & 2 \\
Gingivitis & 8 & 2 \\
Allergy/insect bite & 8 & 2 \\
Dog bite & 8 & 2 \\
Others & 73 & 15 \\
\hline
\end{tabular}

Others: Skin rash, diarrhea, dysmenorrhea, lumbago, trauma, candidiasis, wound, appendicitis, somatic pain, hernia, asthma, burn, oral sores, breast enlargement, pulpitis, measles, dermatitis, severe fibroids, and fatigue.

The most commonly prescribed antibiotics were penicillins (324, 48\%), nitroimidazoles $(108,16 \%)$, quinolones $(58,9 \%)$, cephalosporins $(56,8 \%)$, and aminoglycosides $(47,7 \%)$ (Table 2).

Percentage of encounters with one or more antibiotics prescribed was $23 \%(10,402 / 45,160)$ with 55\% (369/669) of the antibiotics prescribed by generic name. On average, $1.3(669 / 500)$ antibiotics were prescribed per patient and 29\% (144/500) of the patients received two or more antibiotics. Of the total number of patients, $125(25 \%)$ received antibiotic injections. All the medicines prescribed were from the Essential Medicines and Health Supplies List (EMHSL) of Uganda. The average cost of antibiotics prescribed per patient was USD 0.5 . Antibiotics were prescribed for an average duration of 4.8 days (3-10), with majority of patients $440(88 \%)$ receiving antibiotics for a duration of five days. Majority of the prescriptions, 485 (97\%), had diagnosis of the patient recorded. However, $133(27 \%)$ of the 
diagnoses did not comply with the Uganda Clinical Guidelines (UCG) and were, therefore, considered inappropriate. Of the $367(73 \%)$ of the diagnoses that were compliant with the guidelines, $155(42 \%)$ of the antibiotics prescribed for the diagnosis were not compliant with UCG. (Table 3, Supplementary Materials Tables S1-S6).

Table 2. Showing class of antibiotics prescribed.

\begin{tabular}{ccc}
\hline Class of Medicine & Frequency $(\boldsymbol{n = 6 6 9 )}$ & Percentage \\
\hline Penicillins & 324 & 48 \\
Nitroimidazoles & 108 & 16 \\
Fluoroquinolones & 58 & 9 \\
Cephalosporins & 56 & 8 \\
Aminoglycoside & 47 & 7 \\
Sulphonamides & 26 & 4 \\
Tetracyclines & 29 & 4 \\
Macrolides & 16 & 2 \\
Nitrofurantoin & 5 & 1 \\
\hline
\end{tabular}

Penicillins (Amoxycillin 24\%, Penicillin Procaine Fortified 11\%, Ampicillin+amoxicillininj 7\%, Penicillin benzyl $6 \%$ ), Nitroimidazoles (metronidazole 16\%), Fluroquinolones (Ciprofloxaxin 9\%), cephalosporins (Ceftriaxone 8\%), aminoglosides (Gentamycin 7\%), sulfonamides (Cotrimoxazole 4\%), Macrolides (Erythromycin 2\%).

Table 3. Showing results for prescribing indicators.

\begin{tabular}{|c|c|c|c|}
\hline Prescribing Indicators & $\begin{array}{c}n \\
\text { (Percentage/Range) }\end{array}$ & National Standard & WHO Standard \\
\hline Percentage of encounters with one or more antibiotics prescribed & $10,402(23 \%)$ & $\leq 15 \%$ & $20-26 \%$ \\
\hline Percentage of antibiotics prescribed by generic name & $369(55.2 \%)$ & $100 \%$ & $100 \%$ \\
\hline Percentage of patients prescribed antibiotics with diagnosis recorded & $485(97 \%)$ & $85 \%$ & $100 \%$ \\
\hline Percentage of diagnoses compliant with Uganda Clinical Guidelines (UCG) & $367(73 \%)$ & & \\
\hline Percentage of patients receiving antibiotic injections & $125(25 \%)$ & $\leq 15 \%$ & $13.4-24.1$ \\
\hline Percentage of antibiotics prescribed consistent with the EMHL & $669(100 \%)$ & $100 \%$ & $100 \%$ \\
\hline Percentage of prescriptions in accordance with Uganda Clinical Guideline (UCG) & $212(58 \%)$ & $100 \%$ & $100 \%$ \\
\hline Average cost of antibiotics prescribed per patient in USD & $\$ 0.5(0.4-0.8)$ & $\$ 0.5-0.9$ & \\
\hline Average duration of prescribed antibiotic treatment days & $4.8(3-10)$ & $5-10$ & $5-10$ \\
\hline
\end{tabular}

The main reasons for noncompliance of the prescriptions with the guidelines included prescribing antibiotics for malaria (50,32\%); helminthiasis (worm infestation) $(17,11 \%)$; dental conditions $(19,12 \%)$ such as pulpitis and gingivitis, for which antibiotics are not indicated; and noninfectious conditions such as dysmenorrhea and lumbago $(23,15 \%)$ (Table 4). The diagnoses that were considered noncompliant with the guidelines included nonspecific and vaguely written diagnosis such as respiratory tract infections $(59,44 \%)$, upper respiratory tract infections $(40,30 \%)$, gastroenteritis $(15,11 \%)$, and bacterial infection $(10,8 \%)$ (Table 5).

Table 4. Showing diagnosis for which antibiotic prescriptions were considered noncompliant with the guidelines.

\begin{tabular}{ccc}
\hline Diagnosis & Frequency $(\boldsymbol{n}=\mathbf{1 5 5})$ & Percentage \\
\hline Malaria & 50 & 32 \\
Helminthiasis & 17 & 11 \\
Gastritis & 14 & 9 \\
Dental conditions & 19 & 12 \\
Viral conditions & 14 & 9 \\
Candidiasis & 8 & 5 \\
Noninfectious conditions & 23 & 15 \\
Others & 10 & 6
\end{tabular}

Dental conditions included pulpitis, dental caries, and gingivitis; viral conditions were chicken pox, measles, acute diarrhea, common cold, and herpes zoster; noninfectious conditions included dysmenorrhea, lumbago, fatigue, skin allergy/rash, pain, and hernia; others were scabies, wrong antibiotics, or single antibiotic when combination is indicated. 
Table 5. Showing diagnoses that were considered noncompliant with the guidelines.

\begin{tabular}{ccc}
\hline Diagnosis & Frequency $(\boldsymbol{n = 1 3 3 )}$ & Percentage \\
\hline Respiratory tract infections & 59 & 44 \\
Upper respiratory tract & 40 & 30 \\
infections & 15 & 11 \\
Gastroenteritis & 10 & 8 \\
Bacterial infection & 4 & 3 \\
Lower respiratory tract & 5 & 4 \\
infections & 5 Others * &
\end{tabular}

\subsection{Health Facility Factors Affecting Prescribing Practices}

All the facilities had UCG and no EMHSL. Only two (40\%) facilities had diagnostic laboratory facilities for complete blood count, and these were the health center grade IVs (HCIVs). On average, 68\% (50/75) of the antibiotics were available on the day of the visit. No health facility had all the selected antibiotics available on the day of the visit and two injectable antibiotics, penicillin benzyl and penicillin procaine fortified (PPF), were available throughout the review period. The average number of days the antibiotics were out of stock was eight days. Cloxacillin was most frequently out of stock with only one facility that did not register a stock out in the review period. The percentage cost contribution of antibiotics was 38\% (11,904,870 Ugandan shillings, about 3200 USD) of total expenditure on essential medicines (31,673,655 Ugandan shillings, about 8500 USD). The percentage contribution for higher-level facilities ( $35 \%$ and $27 \%$ ) was less than that of lower-level facilities (44\%,51\%, and 56\%). (Table 6, Tables S7 and S8).

Table 6. Showing results for health facility indicators.

\begin{tabular}{|c|c|c|c|}
\hline Health Facility Indicators & $\begin{array}{c}n \\
\text { (Percentage/Range) }\end{array}$ & National Standard & WHO Standard \\
\hline Existence of standard treatment guidelines (STGs/UCG) for infectious diseases & $5(100 \%)$ & $100 \%$ & $100 \%$ \\
\hline Existence of an approved hospital essential medicines list & $0(0 \%)$ & $100 \%$ & $100 \%$ \\
\hline Availability of a set of key antibiotics in the facility stores on the day of the study & $51(68 \%)$ & $100 \%$ & $100 \%$ \\
\hline Average number of days that a set of key antibiotics is out of stock in the review & $8(5-15)$ & $0 \%$ & $0 \%$ \\
\hline Expenditure on antibiotics as a percentage of total facility medicine costs & $\$ 3200(38 \%)$ & & \\
\hline Availability of diagnostic laboratory facility (Complete blood count) & $2(40 \%)$ & $100 \%$ & $100 \%$ \\
\hline
\end{tabular}

\section{Discussion}

In order to design effective interventions to improve appropriate use of antibiotics, it is important to understand prescribing practices of antibiotics. This study provides an understanding of these prescribing practices.

The findings of this survey revealed high percentage of patients receiving antibiotics and low percentage of prescribing by generic name. Studies done on antibiotic prescription patterns in a Ghanaian primary health care facility and Khartoum state found a higher percentage encounter of one or more antibiotics prescribed, of $29 \%$ and $54 \%$, respectively [22,23]. This difference could also be attributed to a limited range of antibiotics available in public health facilities in Uganda. However, our findings are within WHO standard $20-26 \%$ and more than the national standard $\leq 15 \%$ [24-26]. This high exposure to antibiotics means high chances of development of resistance. The prescription by generic name is well below the WHO standard of $100 \%$ and national standard of $\geq 85 \%$ [24-26]. Previous studies conducted in Cameroon and Khartoum state found $98 \%$ and $37 \%$ antibiotics were prescribed by generic names, respectively [22,23]. Prescribing by generic name reduces dispensing errors, promotes patient understanding of medicines, and prevents extravagant prescribing, hence improving medicine use and should, therefore, be encouraged among the prescribers. The average number of antibiotics per prescription was lower than that reported in a study carried out at a tertiary teaching care hospital, Gujrat, of 
1.5 and WHO standard of 1.6-1.8 [25-27]. It is preferable to keep the mean number of antibiotics per prescription as low as possible so as to prevent risk of drug-drug interaction and reduce cost of treatment, pill burden, and out of stock of antibiotics. This will in turn reduce exposure to antibiotics and promote adherence, slowing the development of antibiotic resistance.

The average cost of antibiotics prescribed per patient $(\$ 0.5)$ was higher than per capita allocation for medicines (\$0.3). The expenditure on antibiotic medicines as a percentage of total facility medicine costs was also high (38\%). These show the health facilities operated with small budgets for medicines and spent more on antibiotics. The lower health facilities that receive kits (push system) had higher expenditure on antibiotics compared with higher levels that determine their own needs (pull system). Adding to this expenditure was the high percentage of patients receiving parenteral antibiotics more than the WHO standard for percentage of encounters, with an injection prescribed $13-24 \%$ and national standard of less than or equal to $15 \%$ [24-26]. More so, expensive parenteral antibiotics such as ceftriaxone were also commonly used. Unnecessary use of parenteral antibiotics adds to cost of therapy and also increases the risk of blood-borne infections and other complications associated with use of injections. Therefore, improving antibiotic use, thereby reducing antibiotic consumption, can reduce costs on antibiotics and increase antibiotic availability. The availability of antibiotics was low. No health facility had all the selected antibiotics available. Some of the patients did not have their prescriptions filled if antibiotics were unavailable, hence failure to get treatment. This can lead to resistance, death, or other complications and out-of-pocket expenditure, which the individuals may not afford, being a poor community. Non-availability of antibiotics also leads to loss of confidence by the patients in the health care facility and system and can affect prescribing patterns, as the prescribers may be forced to prescribe what is available but not what is appropriate for the clinical condition.

Adherence to UCG was found to be suboptimal. Recording of diagnosis before prescriptions was above the national target of $85 \%$, though lower than WHO standard of 100\% [24-26]. Respiratory tract infections and urinary tract infections were the main diagnoses for which antibiotics were prescribed. These correlate with the prescribing patterns of the antibiotics. For example, the UCG recommends amoxicillin for non-severe pneumonia and ceftriaxone for severe pneumonia, while ciprofloxacin is recommended for urinary tract infections including acute cystitis and prostatitis. Only $73 \%$ of the antibiotic prescriptions had the diagnosis properly written following UCG despite all the facilities having UCG. The diagnoses that were considered noncompliant with the guidelines included vaguely written and nonspecific diagnoses such as respiratory tract infections/upper respiratory tract infections, bacterial infections, and gastroenteritis. This can affect use of the treatment guidelines, reporting of prevalence/incidence of diseases, and antibiotic use. For example, it is difficult to tell whether respiratory tract infection is viral or bacterial and, therefore, warrants antibiotic or not. More so, each diagnosis in the guidelines has an International Classification of Diseases 10th version (ICD10) code and documentation and reporting are based on this code. The noncompliance may be as a result of knowledge gaps and diagnostic uncertainty and it may also imply that the treatment guidelines are not being referred to while prescribing medicines. In fact, $42 \%$ of the prescriptions did not comply with UCG and only the two higher-level health facilities had diagnostic laboratory equipment for complete blood count. The main reasons for noncompliance of the prescriptions with the guidelines included prescribing antibiotics for malaria, helminthiasis (worm infestation), dental conditions such as pulpitis and gingivitis for which antibiotics are not indicated, and noninfectious conditions such as dysmenorrhea. Following the UCG 2016, Ministry of Health Uganda, no antibiotic medicines should be administered as treatment for those conditions [28]. This demonstrates antibiotic misuse, which can potentially lead to increased antibiotic resistance, thus increasing the necessity to use more expensive antibiotics to treat life-threatening infections caused by resistant bacteria in the future. As observed by Ofori-Asenso and Agyeman, overuse of antibiotics for nonspecific 
conditions such as childhood diarrhea, mild nonbacterial infection, upper respiratory tract infection, and simple malaria contributes to antibiotic resistance [11]. These irrational prescribing patterns may be a result of inadequate knowledge and skills in prescribing antibiotics, lack of diagnostic facilities, and low cadre prescribers. A study conducted in Uganda indicated that the majority of prescribing cadres were lower-cadre professionals such as nursing assistants and enrolled nurses [29]. The lower health facilities without diagnostic laboratory equipment may not be able to appropriately diagnose infections since most of their diagnoses will be based on clinical signs and symptoms. This further perpetuates prescribing to be on the safe side.

As a limitation, these indicators highlight major problem areas of antibiotic use patterns and quantify the magnitude of the problem at glance, but they do not exhaustively answer why the problem existed. The study was also limited only to outpatients and, therefore, could not give overall antibiotic prescribing patterns in the facilities.

\section{Materials and Methods}

This study was conducted in Yumbe district located in the northwestern part of Uganda, located approximately $590 \mathrm{~km}$ from Kampala, Uganda's capital city. The district total population is 485,582 , of which $48 \%$ are males and $52 \%$ are females. The district hosts refugees mainly from South Sudan and the Democratic Republic of Congo. There are 27 health facilities in the district. These include a general hospital (Yumbe hospital), which is the district referral hospital, two HC IVs, seven (7) health center grade IIIs (HC IIIs), and 17 health center grade IIs (HC IIs). These health facilities provide both curative and preventive services including Outpatient, in patient, Maternal and child health services, and community outreaches, plus other specialized services depending on the level of the facility. The study was conducted in five Health facilities: Yumbe Hospital/Yumbe HC IV, Midigo HC IV, Ariwa, Barakala, and Kulikulinga HC IIIs. These Health facilities were purposely selected. They attend to refugees and have admission facilities and laboratories. Yumbe Hospital was under major renovation and its services were transferred to Yumbe HC IV during the data collection period.

This was a cross-sectional study. Outpatient records for a period of three months from March-May 2019 were reviewed. WHO core medicine use indicators for assessing outpatient medicine use were used.

The study population included outpatients who were prescribed antibiotics from the five health facilities and tracer antibiotics.

Prescriptions containing systemic antibiotics for both adults and children were considered for inclusion, while prescriptions containing topical antibiotics such as lotions, ointments, vaginal pessaries, and eye preparations like eye drops were excluded. Also, prescriptions from outreach and special doctors' clinics were excluded. Only the selected 15 tracer antibiotics were used to assess for availability. Antibiotics in this study refer to medicines for treatment of bacterial infections only.

Based on WHO/DAP/93 recommendation for sample size determination and sampling technique for prescribing indicators, 100 prescriptions were taken from each corresponding Health facility [30], meaning a total of 500 prescription forms meeting the inclusion criteria were reviewed in this study. A systematic sampling technique was employed to select the 100 prescriptions from each Health facility. The total numbers of prescriptions in the review period with an antibiotic prescribed were determined and the sampling interval was determined by dividing the total number by 100 . A simple random sampling was used to select the first prescription. For Health facility indicators, 15 key tracer antibiotics were selected from each facility as per WHO recommendation of a minimum of 15 essential tracer medicines in each health facility. These were purposely selected by only including antibiotics that are expected to be available at the lowest level of care of the study facilities, i.e., HC III, according to the national EMHSL.

The study indicators included:

- Prescribing indicators; 
- Percentage of encounters with one or more antibiotics prescribed;

- Percentage of antibiotics prescribed by generic name;

- Average number of antibiotics prescribed per patient;

- Percentage of patients prescribed antibiotic medicines with diagnosis recorded;

- Percentage of patients receiving antibiotic injections;

- Percentage of antibiotics prescribed, consistent with the EMHSL;

- Average cost of antibiotics prescribed per patient;

- Average duration of prescribed antibiotic treatment;

- Percentage of prescriptions in accordance with the Standard Treatment Guidelines (STGs);

- Facility indicators/factors;

- Existence of STGs for infectious diseases;

- Existence of an approved hospital formulary list or essential medicines' list (EML);

- Availability of a set of key antibiotics in the facility stores on the day of the study;

- Average number of days that a set of key antibiotics is out of stock;

- Expenditure on antibiotics as a percentage of total facility medicine costs; and

- Availability of diagnostic laboratory facility (complete blood count).

Data were collected using a structured check list for prescribing and health facility indicators. Data regarding prescribing indicators were taken from sampled prescription records retrospectively and were filled or recorded in the structured check list accordingly. Additionally, the availability of tracer antibiotics, which were assessed from the store, and the presence of EML and STG in the Outpatient Department (OPD) were also assessed in the facility indicator form accordingly. Data were collected from OPD registers, Dispensing logs, and stock cards or stock book. Additional information (e.g., prices) was obtained from recent invoices. Data collection was supervised by the researchers.

Microsoft Excel 2010 version was used for the analysis. The scores of the indicators were compared with national and WHO standards/targets.

Makerere University School of Health Sciences Research Ethics committee (MU-SHSREC) granted ethical approval for the study.

\section{Conclusions}

Irrational prescribing practices observed in this study included a high number of encounters with an antibiotic prescribed, low percentage of prescribing by generic name, higher percentage of patients receiving injectable antibiotics, and low compliance with standard treatment guidelines even though the treatment guidelines where available in all the facilities. Most of the diagnoses did not comply with the treatment guidelines and antibiotics were prescribed for indications such as uncomplicated malaria, helminthiasis, and allergy that do not require antibiotic interventions in their standard management guides. The facility factors that could have affected the prescribing practices included low availability, out-of-stock medicines, and lack of diagnostic laboratory facilities. These can lead to suboptimal therapeutic outcomes and perpetuate development of drug resistance.

There is need to strengthen antibiotic use in health facilities and hospitals through setting up stewardship programs and interventions to enforce the national standard treatment guidelines and provision of diagnostic facilities. More emphasis needs to be placed on teaching the art of writing a prescription to prescribers and compliance with standard treatment guidelines. There is also need to improve availability of the antibiotics. We recommend future studies on the outcomes of antibiotic treatment, underlying causes of irrational antibiotic use, and antibiotic resistance patterns in these facilities.

Supplementary Materials: The following are available online at https:/ /www.mdpi.com/2079-638 2/10/2/172/s1. Table S1: Percentage of encounters with one or more antibiotics prescribed. Table S2: Percentage of antibiotics prescribed by generic name. Table S3: Average number of antibiotics prescribed per patient. Table S4: Percentage of encounters with antibiotic injection prescribed. Table S5: Average cost of antibiotics prescribed per patient. Table S6: Average duration of prescribed antibiotic treatment. Table S7: Availability of a set of key antibiotics in the facility stores on the day of the study. Table S8: Average number of days that a set of key antibiotics is out of stock. 
Author Contributions: Conceptualization, M.B. and K.R.; Data curation, M.B. and K.R.; Formal analysis, M.B., W.N., and K.R.; Investigation, M.B. and K.R.; Methodology, M.B. and K.R.; Project administration, M.B.; Resources, M.B.; Supervision, K.R.; Validation, M.B., W.N., and K.R.; Visualization, M.B., W.N., and K.R.; Writing—original draft, M.B., W.N., and K.R.; Writing-review and editing, M.B., W.N., and K.R. All authors have read and agreed to the published version of the manuscript.

Funding: This research received no external funding.

Data Availability Statement: The data for the study will be available upon reasonable request.

Acknowledgments: We acknowledge that the East African Community (EAC) German Development Bank (KFW) funded Masters Scholarship Program that funded the course of Health Supply Chain Management (HSCM) at Regional Center of Excellence for Vaccines, Immunization and Health supply chain management (RCE-VIHSCM) in University of Rwanda that led us to conduct this research as a requirement of the program. We also acknowledge the team at RCE-VIHSCM in University of Rwanda for all the support and guidance during the conduct of the study.

Conflicts of Interest: The authors declare no conflict of interest.

\section{References and Note}

1. Prajapati, V.; Bhatt, J. Evaluation of Rational Usage of Antimicrobial Agents in the Paediatric Department at Tertiary Teaching Care Hospital, Gujrat. Int. J. Pharm. Sci. Res. 2014, 5, 5374.

2. WHO. No Time to Wait: Securing the Future from Drug-Resistant Infections; Report to the Secretary General of the Nations; WHO: Geneva, Switzerland, 2019.

3. Unas, C. Antibiotic Resistance in Uganda: Situation Analysis and Recommendations; Uganda National Academy of Sciences, Center for Disease Dynamics, Economics \& Policy: Kampala, Uganda, 2015; p. 107.

4. Mpairwe, Y.; Wamala, S. Antibiotic Resistance in Uganda: Situation Analysis and Recommendations; Uganda National Academy of Sciences: Kampala, Uganda, 2015.

5. Lesho, E.P.; Laguio-Vila, M. The slow-motion catastrophe of antimicrobial resistance and practical interventions for all prescribers. In Mayo Clinic Proceedings; Elsevier: Amsterdam, The Netherlands, 2019.

6. Holmes, A.H.; Moore, L.S.P.; Sundsfjord, A.; Steinbakk, M.; Regmi, S.; Karkey, A.; Guerin, P.J.; Piddock, L.J.V. Understanding the mechanisms and drivers of antimicrobial resistance. Lancet 2016, 387, 176-187. [CrossRef]

7. Anong, D.N.; Akoachere, J.-F.K. Prescribing patterns and associated factors of antibiotic prescription in primary health care facilities of Kumbo East and Kumbo West Health Districts, North West Cameroon. PLoS ONE 2018, 13, e0196861.

8. Kotwani, A.; Wattal, C.; Katewa, S.; Joshi, P.C.; Holloway, K. Factors influencing primary care physicians to prescribe antibiotics in Delhi India. Fam. Pract. 2010, 27, 684-690. [CrossRef]

9. Chauhan, I.; Yasir, M.; Kumari, M.; Verma, M. The pursuit of rational drug use: Understanding factors and interventions. Pharmaspire 2018, 10, 48-54.

10. World Health Organization. Promoting Rational Use of Medicines: Core Components; World Health Organization: Geneva, Switzerland, 2002.

11. Ofori-Asenso, R.; Agyeman, A.A. Irrational Use of Medicines-A Summary of Key Concepts. Pharmacy 2016, 4, 35. [CrossRef]

12. Salgado, F.X.C.; Sanchez, T.E.G.; Da Silva, N.B.; Oliveira, H.B.; Karnikowski, M.; Karnikowski, M.G.D.O. Evaluation of rational use of antimicrobial agents in a Brazilian intensive care unit. Health 2014, 6, 188-194. [CrossRef]

13. Holloway, K.; Dijk, L.V. Rational Use of Medicines; World Health Organization: Geneva, Switzerland, 2011.

14. Hillman, A. Cost-effectiveness opportunities for new antibiotics. PharmacoEconomics 1994, 5, 40-43. [CrossRef]

15. Col, N.F.; O'Connor, R.W. Estimating worldwide current antibiotic usage: Report of Task Force 1. Rev. Infect. Dis. 1987, 9, 232-243. [CrossRef]

16. Obua, C.; Ogwal-Okeng, J.W.; Waako, P.; Aupont, O.; Ross-Degnan, D. A Comparison of Prescribing Practices in Public and Private Sectors in Uganda.

17. Ogwal-Okeng, J.W.; Obua, C.; Waako, P.; Aupont, O.; Ross-Degnan, D. A comparison of prescribing practices between public and private sector physicians in Uganda. East Afr. Med. J. 2004, 2004, S12-6.

18. Ucakacon, P.S.; Achan, J.; Kutyabami, P.; Odoi, A.R.; Kalyango, N.J. Prescribing practices for malaria in a rural Ugandan hospital: Evaluation of a new malaria treatment policy. Afr. Health Sci. 2011, 11, 53-59.

19. Means, A.R.; Weaver, M.R.; Burnett, S.M.; Mbonye, M.K.; Naikoba, S.; McClelland, R.S. Correlates of Inappropriate Prescribing of Antibiotics to Patients with Malaria in Uganda. PLoS ONE 2014, 9, e90179. [CrossRef]

20. Kibuule, D.; Kagoya, H.R.; Godman, B. Antibiotic use in acute respiratory infections in under-fives in Uganda: Findings and implications. Expert Rev. Anti Infect. Ther. 2016, 14, 863-872. [CrossRef]

21. Mcgaughey, N.; Lynch, M.; Bell, D. Prescribing practices and polypharmacy in Kitovu Hospital, Uganda. East Cent. Afr. J. Pharm. Sci. 2010, 13, 66-71.

22. Mahmoud, R.; Ali, H.; Kheder, S. Prescribing rationality in Khartoum state, Sudan: An update. Sudan Med. Monit. 2014, 9, 61. [CrossRef] 
23. Prah, J.; Kizzie-Hayford, J.; Walker, E.; Ampofo-Asiama, A. Antibiotic prescription pattern in a Ghanaian primary health care facility. Pan Afr. Med. J. 2017, 28, 214. [CrossRef] [PubMed]

24. Trap, B.; Ladwar, D.O.; Oteba, M.O.; Embrey, M.; Khalid, M.; Wagner, A.K. Article 1: Supervision, Performance Assessment, and Recognition Strategy (SPARS)_A multipronged intervention strategy for strengthening medicines management in Uganda: Method presentation and facility performance at baseline. J. Pharm. Policy Pr. 2016, 9, 1-15. [CrossRef] [PubMed]

25. Desalegn, A.A. Assessment of drug use pattern using WHO prescribing indicators at Hawassa University teaching and referral hospital, south Ethiopia: A cross-sectional study. BMC Health Serv. Res. 2013, 13, 1-170. [CrossRef] [PubMed]

26. Amaha, N.D.; Weldemariam, D.G.; Abdu, N.; Tesfamariam, S. Prescribing practices using WHO prescribing indicators and factors associated with antibiotic prescribing in six community pharmacies in Asmara, Eritrea: A cross-sectional study. Antimicrob. Resist. Infect. Control. 2019, 8, 163. [CrossRef]

27. Prajapati, V.; Bhatt, J. Evaluation of Rational Usage of Antimicrobial Agents in the Medicine Department at Tertiary Teaching Care Hospital, Gujrat. Int. J. Pharm. Sci. Res. 2014, 5, 865.

28. Ministry of Health of Uganda. Uganda Clinical Guidelines 2016; Ministry of Health of Uganda: Kampala, Uganda, 2010.

29. Oteba, M.; Wagner, A.K.; Seru, M.; Embrey, M.; Trap, B. Pharmaceutical system strengthening in Uganda: Implementing a holistic, evidence-informed, long-term strategy. J. Pharm. Policy Pract. 2018, 11, 23. [CrossRef] [PubMed]

30. WHO. How to Investigate Drug Use in Health Facilities: Selected Drug Use Indicators; World Health Organization: Geneva, Switzerland, 1993. 